\title{
EXPERIENCIA DE ESCUELAS SALUDABLES CON POBLACIÓN INDÍGENA Y AFROCOLOMBIANA EN EL DEPARTAMENTO DE CHOCÓ
}

\author{
${ }^{1}$ Andrea Hernández Quirama, ${ }^{2}$ Blanca Patricia Mantilla Uribe, ${ }^{3}$ Alba Yaneth Rincón Méndez, \\ ${ }^{4}$ María Constanza Hakspiel Plata, ${ }^{5}$ Claudia Milena Velasco Rangel, ${ }^{6}$ María del Pilar Oviedo Cáceres

\begin{abstract}
${ }^{1}$ Trabajadora social. Doctora en Investigaciones Feministas y Género. Instituto PROINAPSA. Universidad Industrial de Santander. Bucaramanga, Colombia ${ }^{2}$ Enfermera. Mg. en Pedagogía. Directora Instituto PROINAPSA Universidad Industrial de Santander, Bucaramanga, Colombia

${ }^{3}$ Odontóloga. Mg. en Desarrollo Humano y Sustentable. Instituto PROINAPSA - Universidad Industrial de Santander, Bucaramanga, Colombia

${ }^{4}$ Enfermera. Especialista en Educación y Orientación Sexual. Instituto PROINAPSA - Universidad Industrial de Santander, Bucaramanga, Colombia

${ }^{5}$ Enfermera. Candidata a Mg. en Pedagogía. Instituto PROINAPSA. Universidad Industrial de Santander. Bucaramanga, Colombia

${ }^{6}$ Optómetra. Mg. Salud Pública. Mg. Educación para la Salud. Instituto PROINAPSA. Universidad Industrial de Santander. Bucaramanga, Colombia
\end{abstract}

Autor responsable de correspondencia: Andrea Hernández Quirama:

Correo electrónico: ahernandezq@hotmail.com.

\section{RESUMEN}

Objetivo: se realizó un estudio descriptivo de tipo exploratorio, que buscó dar cuenta de las condiciones de implementación de la estrategia Escuelas Saludables en las instituciones educativas del departamento de Chocó y elaborar un modelo de la estrategia adaptada para población indígena y afrocolombiana.

Materiales y métodos: se hizo un proceso participativo, dividido en dos momentos: recolección, procesamiento y análisis de información que aportó elementos para la construcción del modelo.

Resultados: se diseñó de manera participativa el modelo de Estrategia de Escuelas Saludables para población indígena y afrocolombiana, el cual propone dos rutas, una para las instituciones educativas y otra para generar alianzas intersectoriales e interinstitucionales para la promoción de escuelas saludables, cada ruta contiene cinco pasos; adicionalmente se capacitó a las personas participantes en el modelo propuesto.

Conclusiones: la Estrategia Escuelas Saludables aporta elementos fundamentales para el desarrollo humano, para la promoción de la salud en el ámbito escolar y, por ende, para el mejoramiento de la calidad de vida de las comunidades indígenas y afrocolombianas. [Hernández A, Mantilla BP, Rincón AY, Hakspiel M,C Velasco CM, Oviedo M. Experiencia de escuelas saludables en población indígena y afrocolombiana en el departamento de Chocó. Ustasalud 2015;14:25-31].

Palabras clave: Grupos étnicos, promoción de la salud, escuelas.

\section{EXPERIENCE OF HEALTHY SCHOOLS IN INDIGENOUS AND AFRO-COLOMBIAN POPULATION IN CHOCÓ}

\section{ABSTRACT}

Objective: a descriptive study of exploratory, who sought to account for the conditions of implementation of the Healthy Schools strategy in educational institutions of Choco Department and develop a model adapted to indigenous and Afro-Colombian population strategy was performed.

Methods: collection, processing and analysis of information provided elements for the construction of the model: a participatory process, divided into two moments was performed.

Results: strategy model Healthy Schools for indigenous and Afro-Colombian population, which proposes two routes one for educational institutions and another to generate intersectoral and interagency partnerships to promote healthy schools, each route contains five steps are designed in a participatory manner; additionally it was trained the participants in the proposed model.

Conclusions: the Healthy Schools Strategy provides fundamental for human development, for health promotion in schools and thus to improve the quality of life of indigenous and Afro-Colombian communities elements.

Key words: Ethnic groups, health promotion, schools.

Recibido para publicación: 13 de julio de 2015. Aprobado para publicación: 5 de octubre de 2015. 


\section{INTRODUCCIÓN}

La estrategia Escuelas Saludables ${ }^{1}$ es un lineamiento nacional que pretende o contribuye a integrar las acciones de diferentes sectores e instituciones tendientes a mejorar el entorno familiar y escolar y por ende la calidad de vida de las familias y comunidades educativas. Dicha estrategia ha dado paso a la existencia de la Red Nacional de Escuelas Saludables, que tiene como propósito el intercambio de conocimientos y experiencias de promoción de la salud en el ámbito escolar. En Colombia se cuenta con experiencias de trabajo relacionado con esta estrategia ${ }^{2,3}$, que han servido para orientar su proceso de implementación en las instituciones educativas.

Así mismo, existen experiencias documentadas de la implementación de la Eestrategia en el país ${ }^{4,5}$, pero no se encuentra evidencia escrita sobre procesos llevados a cabo con esta en población indígena o afrocolombiana, sin embargo, se han realizado estudios y trabajos donde se incluyen estudiantes indígenas ${ }^{6-8}$. En este sentido, este artículo presenta la experiencia de un proyecto cuyo objetivo consistió en desarrollar un modelo para la implementación y seguimiento de la estrategia Escuelas Saludables con dicha población en el departamento de Chocó.

\section{MATERIALES Y MÉTODOS}

Se presentaron dos momentos para la adaptación del modelo de la estrategia Escuelas Saludables en el contexto del departamento de Chocó:

1. Recolección, procesamiento y análisis de la información. Como técnicas de recolección de la información se utilizaron tres cuestionarios que buscaron indagar sobre la temática desde varias perspectivas: desde el ámbito de las secretarías de Salud y Educación departamental y municipal, así como desde los docentes indígenas y afrocolombianos. Con estos instrumentos se realizó una línea de base departamental y municipal, se analizaron las condiciones generales de salud integral en la institución educativa y evaluó la institución educativa desde el enfoque de promoción de la salud.

Los instrumentos permitieron captar las percepciones, vivencia y opiniones sobre la implementación de la estrategia en las realidades de estas comunidades, así como los elementos, que de acuerdo con su experiencia, pueden estar afectando significativamente su desarrollo en el departamento. La información obtenida fue analizada y categorizada.
2. Con la información obtenida en el paso anterior, se contó con el insumo para construir de manera participativa un modelo para la implementación y seguimiento de la estrategia Escuelas Saludables, adaptada de acuerdo con las realidades propias de las instituciones y del contexto del Departamento.

En total se contó con la participación de 26 docentes indígenas y afrocolombianos de los municipios de Itsmina y Quibdó, respectivamente y 12 representantes de los sectores salud y educación del Departamento. El proceso se llevó a cabo en el período comprendido entre abril y noviembre de 2011, las personas fueron contactadas a través de convocatoria directa por las secretarías de Salud y Educación. Para realizar el proceso se les explicó los objetivos y los resultados esperados, se pidió la participación voluntaria de las personas que representaban las instituciones educativas y secretarías de Salud y Educación, aclarando su derecho de retirarse en cualquier momento del proceso, respetando las normas de investigación en sujetos humanos estipuladas por la declaración de Helsinki II y la Ley 008430 de 1993 del Ministerio de Salud de Colombia, por la cual se establecen las normas científicas para la investigación en salud.

\section{RESULTADOS}

Teniendo en cuenta el abordaje realizado, se presentan a continuación los resultados del primer momento, de acuerdo con los actores participantes: docentes y representantes de secretarías departamentales de Salud y Educación.

El proceso realizado permitió identificar dificultades para la implementación de la estrategia desde las instituciones educativas, así como desde las secretarías de Salud y Educación departamentales. Tal y como se observa en la Figura 1, se cuenta con equipos desde ambos sectores que coordinan con las instituciones educativas las acciones que se van a realizar; sin embargo, cada uno lo hace con una planeación distinta, lo que denota debilidades en la coordinación interinstitucional.

Desde las instituciones educativas se identifica un panorama complejo en cuanto a diversas dimensiones, se resalta el hecho de que no cuentan con las áreas necesarias, ni baterías sanitarias, espacios deportivos, tanque de almacenamiento de agua, elementos de aseo, salones para los procesos de enseñanza-aprendizaje. De la misma manera, tienen deficiencias en capacitación y en programas de complementación nutricional. En las instituciones indígenas hay carencias en la alimentación de las y los escolares. 


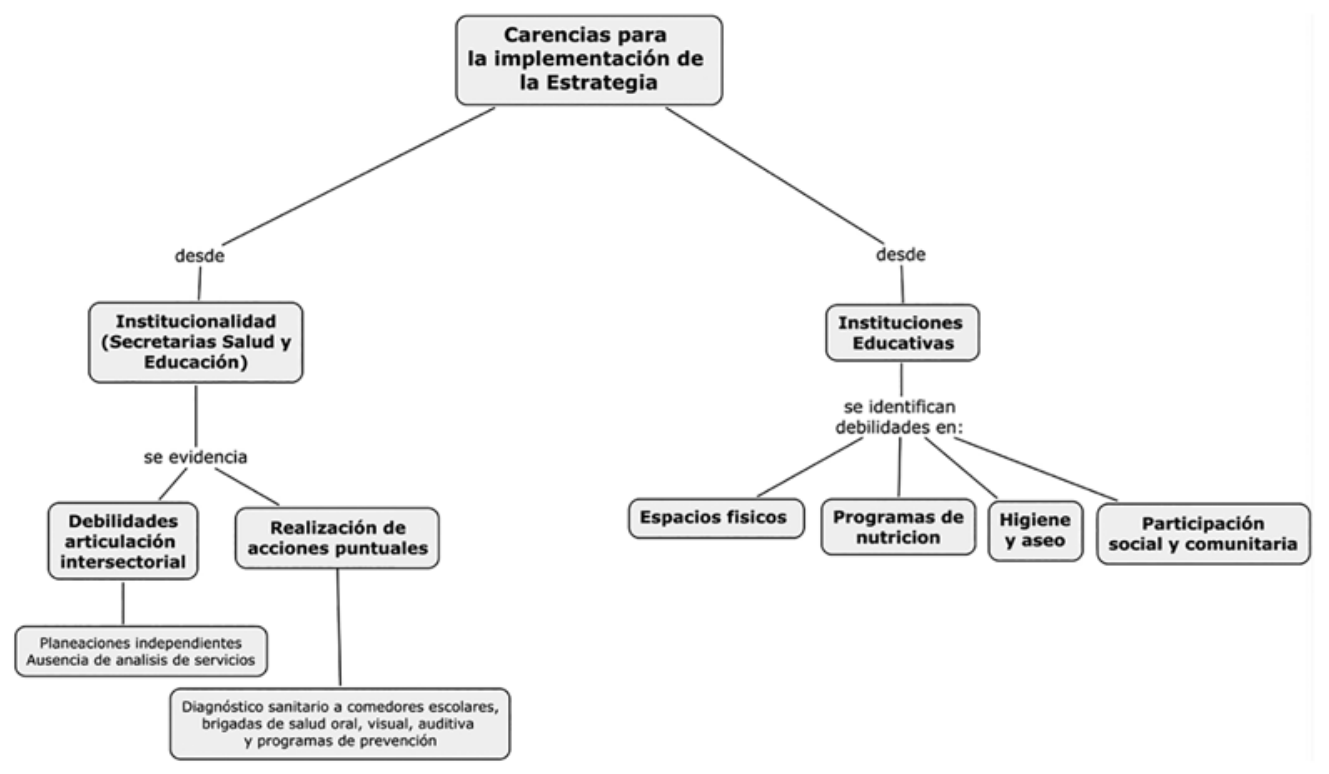

Fuente: Elaboración propia.

Figura 1. Carencias para la implementación de la Estrategia en el Departamento de Chocó.

En cuanto a hábitos higiénicos, en las instituciones educativas afrocolombianas, las aulas no cuentan con elementos de aseo. En las instituciones indígenas se han hecho capacitaciones sobre el tema, pero no tienen rutinas de lavado de manos, pues además de no contar con elementos de aseo, no tienen agua.

En el componente de aseo general, las instituciones educativas afrocolombianas expresaron que no clasifican la basura, no tienen sitio para su almacenamiento, y las cestas para la recolección no tienen tapas, no realizan acciones orientadas al control y erradicación de plagas y vectores. En las instituciones indígenas no tienen baños, cestas de aseo, los residuos se arrojan en un espacio destinado por la comunidad, están quemando la basura, pese a que hay vectores, no hay control de plagas.

En la participación social y comunitaria, en las instituciones educativas afrocolombianas e indígenas se propician espacios de participación en diversas actividades, sin embargo, no se cuenta con instituciones que apoyen las acciones en salud, ni se trabaja con líderes comunitarios.

En el componente de aspectos psicosociales en las instituciones educativas de Quibdó, se realizan actividades de recreación, educación para la sexualidad y convivencia. En las instituciones de Istmina, por medio de la cultura se promueve la convivencia y la participación.

En cuanto a los servicios de salud y bienestar, en las instituciones educativas de Quibdó no disponen de servicios públicos como agua, alcantarillado y siste- ma de recolección de basuras, estas son arrojadas al río, que es su principal fuente de agua. En las instituciones de Istmina no tienen servicios públicos y el agua se recoge del río.

Evaluación institucional desde el enfoque de promoción de la salud

La evaluación permitió a las instituciones educativas definir sus alcances y posibilidades de convertirse en Escuela Promotora de la Salud, desde tres ámbitos de gestión que son: institucional, aula y contexto, la información encontrada se describe a continuación:

Ámbito de gestión institucional. Existen grupos de trabajo que solo se reúnen para aspectos puntuales. No cuentan con políticas escolares definidas. No cuentan con plan de acción para mejorar la salud de los escolares. No hacen procesos de formación a docentes.

Ámbito de gestión de aula. Han realizado actividades de manera esporádica, no cuentan con materiales educativos.

Ámbito de gestión de contexto. No han hecho identificación de situaciones prioritarias que requieren ser intervenidas con ayuda de otros sectores o instituciones.

El segundo momento se caracterizó por concertar y adaptar, de manera participativa con docentes y representantes de las secretarías de Educación y Salud en el ámbito departamental y municipal, el modelo para la implementación y seguimiento de la 
estrategia "Escuelas Saludables", que se desarrolló en dos partes, una "las instituciones educativas", y la segunda, una propuesta metodológica para "generar alianzas intersectoriales e interinstitucionales", para la promoción de escuelas saludables, cada una a través de cinco pasos, los cuales se muestran en la Figura 2.

A continuación se explicita cada ruta de acuerdo con sus pasos.

Pasos para la implementación y seguimiento de la estrategia Escuelas Saludables en las instituciones educativas:

Primer paso. Conformar un equipo gestor. Hace referencia a un equipo líder en la institución educativa con el compromiso individual y colectivo para implementar procesos de promoción de la salud y desarrollo, con espacios de participación real y efectiva que les permita establecer vínculos con directivos, docentes, estudiantes, familias y demás sectores e instituciones de salud, desarrollo social, planeación, universidades, organizaciones no gubernamentales, entre otros, que faciliten el proceso de Escuelas Saludables.
Segundo paso. Reflexionar a la luz del Proyecto Educativo Institucional (PEI) y del Proyecto Etnoeducativo Comunitario (PEC). Revisión que permita analizar grupalmente el PEI y PEC identificando en qué medida hacen aportes a la promoción de la salud.

Tercer paso. Evaluar inicialmente la institución desde el enfoque de Promoción de la Salud. Para identificar los aspectos necesarios por modificar o fortalecer en la institución educativa en: la gestión institucional, de aula y de contexto.

En primer lugar, las acciones que competen a la Gestión institucional y que deben tenerse en cuenta al realizar la autoevaluación son: creación y funcionamiento del equipo gestor; establecimiento de políticas escolares a favor de la salud; análisis de factores relacionados con la salud en la institución educativa; diseño, ejecución y evaluación del plan de acción que favorezca la salud de la comunidad educativa; formación de docentes en aspectos relacionados con salud y que se articulen al PEI y al PEC mediante procesos que han de ser sostenidos en el tiempo; acciones que promuevan la salud de directivos y docentes.

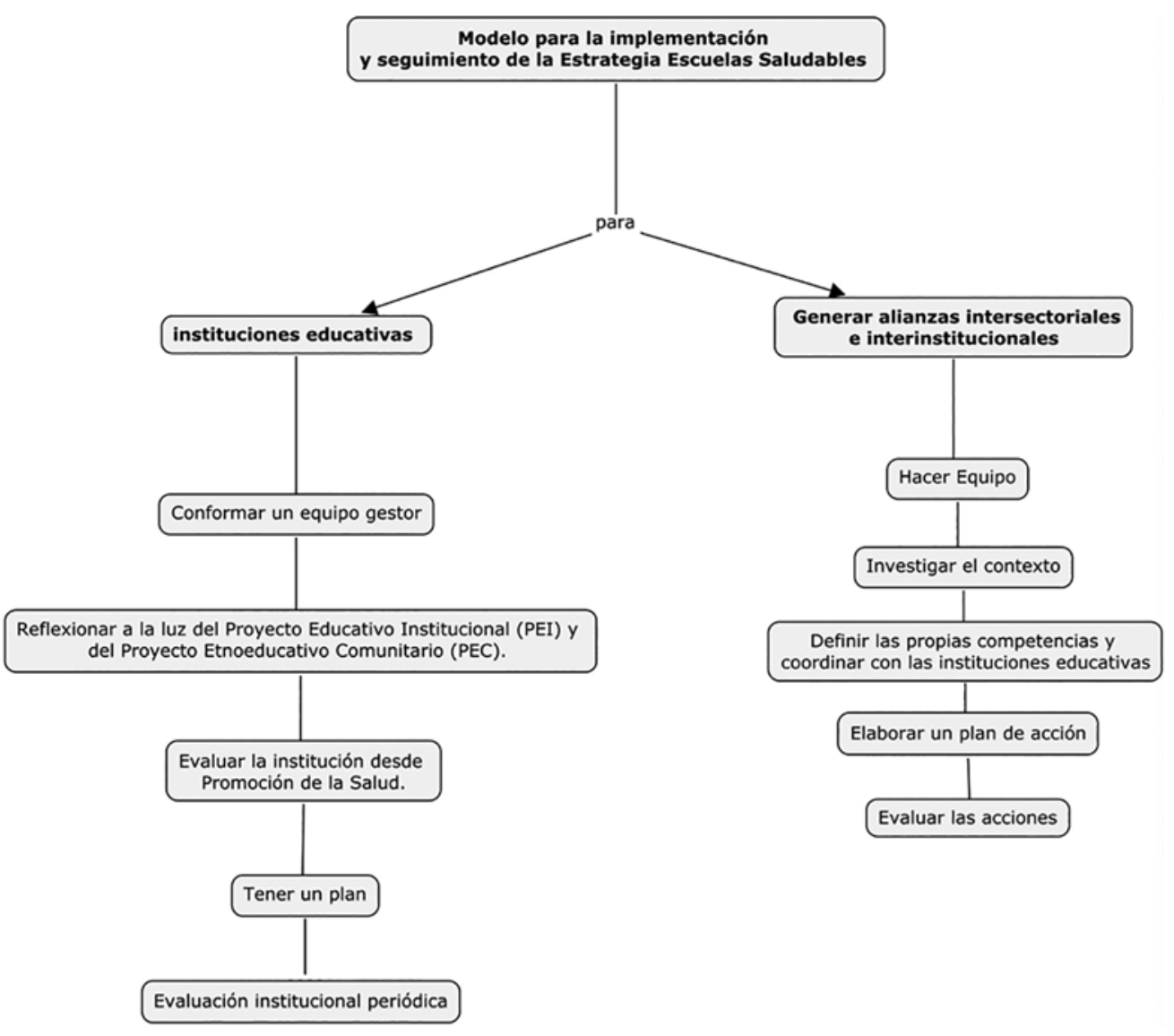

Fuente: Elaboración propia.

Figura 2. Modelo para la implementación de la estrategia Escuelas Saludables. 
En segundo lugar, en la Gestión de aula las acciones para evaluar son: desarrollo de un enfoque pedagógico que favorece la salud, inclusión en el currículo de temáticas específicas de salud integral, y uso de materiales educativos.

En tercer lugar, en la Gestión de contexto, identificar cómo se encuentra el trabajo en cuanto a la integración de la comunidad, sectores e instituciones en aspectos prioritarios para la salud y la educación.

Cuarto paso. Tener un plan. Se sugieren primero, "realizar un análisis de las condiciones generales de salud integral en la institución educativa, en relación con su situación geográfica, la comunidad educativa que la conforma y los aspectos socioeconómicos de las familias". Segundo, "análisis de las condiciones de salud en la institución educativa", teniendo en cuenta: espacios físicos adecuados para el proceso enseñanza-aprendizaje, nutrición, hábitos higiénicos, aseo general, participación social y comunitaria, aspectos psicosociales y servicios de salud y bienestar. Tercero, con base en el análisis de los resultados, se procede a "priorizar" con el fin de concentrar los esfuerzos en la solución de las situaciones más importantes y lograr mejores resultados en el proceso.

Realizado el análisis y la priorización, la institución educativa puede proponer acciones para abordar las situaciones de salud identificadas en cada componente, a través de proyectos articulados con el plan de mejoramiento institucional, así se tendrá insumos para elaborar el plan de acción.

Para realizarlo, se sugiere al equipo gestor tener en cuenta los ámbitos de gestión y las cinco estrategias de promoción de la salud en el ámbito escolar, para elaborar el plan de acción de manera integral y contemplar los elementos que debe tener una Escuela Saludable; las siguientes preguntas orientadoras pueden aportar elementos de análisis para cada uno de los componentes priorizados: ¿Qué podemos hacer mediante políticas escolares saludables?, ¿qué aspectos relacionados con estas situaciones debemos incluir en el currículo?, ¿qué podemos solucionar con ayuda de las entidades externas, los padres, las madres y la comunidad en general?, ¿qué aspectos relacionados con el ambiente debemos transformar para contribuir a la solución de las situaciones de salud identificadas?

Una vez se respondan las preguntas propuestas, el equipo gestor procederá a elaborar el plan de acción para las situaciones identificadas en cada componente y definirá los resultados estratégicos, las metas y las actividades por desarrollar en los ámbitos de gestión institucional, el aula y el contexto. Así se garantiza que las acciones llevadas a cabo, sean evaluadas posteriormente y también se puedan mejorar.

Quinto paso. Evaluación institucional periódica. Requiere ser realizada en diferentes ámbitos. Primero, la evaluación del plan de trabajo, implica el diseño de un sistema de evaluación y seguimiento del plan de trabajo para recopilar evidencia objetiva de los resultados. A partir de esta evaluación, el plan de mejoramiento se enriquece anualmente. Esta es una oportunidad para que las instituciones educativas identifiquen mejores formas de desarrollar su quehacer y las hagan realidad.

Segundo, la evaluación periódica de la institución educativa desde el enfoque de promoción de la salud, se sugiere que anualmente se realice para modificar aquellos aspectos que lo requieran. Lo cual aportara insumos para realizar nuevos proyectos y acciones que el equipo gestor y la comunidad educativa consideren más pertinentes para seguir promoviendo la salud en la institución educativa.

La segunda parte comprende la propuesta metodológica para generar alianzas intersectoriales e interinstitucionales para la promoción de escuelas saludables, se trata de otra ruta para que las distintas entidades y sectores se organicen y trabajen en torno a la salud de los escolares.

Se propone una ruta para todos los sectores y es la clave para que se conviertan en aliados de las instituciones educativas en el camino de la promoción de la salud. La entrada de los aliados en la comunidad educativa debe hacerse al ritmo de las necesidades de cada institución y a la luz de los hallazgos que genere el aporte de otros sectores, a continuación se presentan los cinco pasos propuestos:

Primer paso. Hacer equipo: con representantes de diferentes sectores e instituciones que coordinen y dinamicen el proceso, un equipo líder que ponga el plan en funcionamiento y lo mantenga, debe estar conformado por diferentes sectores e instituciones, es importante que sea interdisciplinario, dinámico y que facilite los procesos.

Algunas de las funciones del equipo pueden ser: realizar un análisis de las condiciones de salud y bienestar de los escolares y actualizarlo en forma periódica; elaborar el plan para la gestión y ejecución de servicios de salud y bienestar para las instituciones educativas; establecer los convenios y acuerdos institucionales; realizar un proceso de seguimiento para verificar las acciones y determinar si se han producido avances en el desarrollo del plan. 
Segundo paso. Investigar el contexto: se propone recopilar información que facilite el análisis general de las condiciones de salud de las instituciones educativas, como: número, tipo (privada, pública); ubicación geográfica; características sociodemográficas de la población escolar, población en edad escolar desplazada por el conflicto armado interno, cobertura de seguridad social en salud de escolares; principales causas de morbilidad y mortalidad; diagnóstico nutricional de niños y niñas, número de casos de maltrato infantil, consumo sustancias psicoactivas, de alcohol y tabaco, reporte de casos identificados en salud visual, auditiva y oral de escolares, coberturas poblacionales, porcentaje de retención, de repetición, de ausentismo escolar, de deserción escolar, programas o proyectos de salud, condiciones de las estructuras físicas y entorno ambiental.

La información será insumo para la formulación e implementación de los planes de acción y en la promoción de los proyectos de salud y bienestar como parte de las acciones que conducen al desarrollo de los pueblos indígenas y afrocolombianos.

Tercer paso. Definir las propias competencias y coordinar con las instituciones educativas: para lograr la articulación con las instituciones educativas se sugiere que cada sector defina sus propias competencias y a su vez coordinen el desarrollo de acciones que requiere trabajo articulado.

Partiendo de la premisa que las competencias de cada sector se complementan con las acciones pedagógicas y de gestión que desarrollan las instituciones educativas, se propone que una vez los sectores hayan identificado cuál es su injerencia en la salud y el bienestar de la población escolar, y después del análisis realizado, el equipo de trabajo realice el siguiente proceso:

- Acercamiento y socialización con las directivas de las instituciones educativas.

- Identificación de las situaciones de salud que afectan o son un factor de riesgo para la población escolar y que deben ser intervenidas.

- Definición de las causas que están generando las situaciones de salud.

- Priorización de situaciones que se puedan fortalecer conjuntamente en el marco del desarrollo integral a favor de la salud de la población escolar.

- Elaboración conjunta de un plan de intervención con acciones, objetivos, metas, responsables e indicadores de evaluación de las acciones.

Cuarto paso. Elaborar un plan de acción: para realizarlo se recomienda tener en cuenta la investigación del contexto realizada, los planes de desarrollo de cada sector u organización participante y las necesidades priorizadas en cada institución educativa con la que se hayan hecho alianzas.

Se proponen las siguientes acciones como una forma de apoyar los procesos en las escuelas saludables: Formulación de políticas públicas saludables dirigidas a la población escolar; apoyo a los procesos de educación para la salud dirigidos a personal docente e implementación y fortalecimiento de servicios de salud y bienestar dirigidos al estudiantado.

Se requiere un trabajo articulado y participativo de los diferentes sectores, en este sentido se propone construir rutas de atención que deben contemplar aspectos, como: Identificación de las necesidades de salud en la institución educativa; Definición del proceso para satisfacer dichas necesidades; Definición de actores responsables de la satisfacción del derecho y divulgación de la ruta.

Quinto paso. Evaluar las acciones: el equipo aliado definirá cuáles metodologías e instrumentos aplicará para hacer seguimiento y evaluación de las acciones realizadas.

Finalmente se hizo un proceso de capacitación con docentes y directivos de las instituciones educativas participantes y representantes de las secretarías de Salud y Educación departamental y municipal, sobre la implementación del modelo de la estrategia Escuelas Saludables, donde por una parte se presentó la propuesta metodológica para abordar la promoción de la salud en las instituciones educativas, y por otra, se dio a conocer la ruta para generar alianzas intersectoriales e interinstitucionales.

\section{CONCLUSIONES}

Para que los pueblos indígenas y afrocolombianos gocen de salud integral, se necesita la compañía de diversos actores para lograr la promoción de la salud y el desarrollo humano. En este sentido, debe tenerse en cuenta la heterogeneidad étnica y cultural de las instituciones educativas y la importancia de identificar formas innovadoras en la atención, para favorecer la salud y educación intercultural. De esta manera se responderá a las necesidades de las instituciones educativas con propuestas enmarcadas dentro de los proyectos educativos institucionales que cada una tenga.

Diseñar el modelo de estrategia Escuelas Saludables para población indígena y afrocolombiana implicó un ejercicio de revisión de etnoeducación y salud intercultural, que se fortaleció con el trabajo en terreno con la población, y aportó elementos claves para realizar las adaptaciones necesarias a la realidad de las comunidades con las que se trabajó. 


\section{ARTÍCULO DE INVESTIGACIÓN CIENTÎFICA Y TECNOLÓGICA}

El modelo presentado para la implementación de la estrategia Escuelas Saludables - EES, elaborado y concertado, tuvo acogida por las personas que participaron en el proceso, considerando que esta estrategia es importante abordarla y trabajarla de forma integral en sus respectivas instituciones.

Los docentes participantes manifestaron que consideraban importante la EES, y que era necesario trabajarla con las comunidades indígenas y afrocolombianas, pues les permite conocer la realidad de otras instituciones e intercambiar saberes y acciones y fortalecer sus instituciones educativas como promotoras de la salud en el ámbito escolar.

La estrategia Escuelas Saludables aporta elementos fundamentales para el desarrollo humano, para la promoción de la salud en el ámbito escolar y, por ende, para el mejoramiento de la calidad de vida de las comunidades indígenas y afrocolombianas.

\section{Agradecimientos}

A las instituciones educativas indígenas y afrocolombianas participantes, a las secretarías de Educación y Salud departamental y municipal, a la doctora Lucila Niño por la revisión y aportes realizados al artículo.

\section{REFERENCIAS}

1. Organización Panamericana de la Salud, Ministerio de la Protección Social, Ministerio de Educación Nacional, Ministerio de Ambiente, Vivienda y Desarrollo Territorial. Lineamientos nacionales para la aplicación y el desarrollo de las estrategias de Entornos Saludables, Escuela Saludable y Vivienda Saludable. 2006, Bogotá. URL disponible en: http://www.bvsde.paho.org/texcom/nutricion/ lineam_entornos.pdf
2. Mantilla BP et al. De par en par. Escuelas promotoras de la salud y el desarrollo. Bucaramanga: Publicaciones UIS, 2009.

3. Mantilla BP, Hakspiel MC et al. Hacia la construcción de Escuelas Saludables. Guía para docentes y personal de salud. Bucaramanga: Universidad Industrial de Santander (PROINAPSA-UIS), 2003.

4. Leal Carrillo R, Peña Hernández AC, Reyes Cifuentes P. Evaluación de la estrategia Escuelas Saludables, municipio de Villavicencio 2006-2007. Investigación en Enfermería: Imagen y Desarrollo. 2010;12:95-115.

5. Gutiérrez AM, Gómez OL. Evaluación de proceso de la estrategia Escuelas Saludables en la zona urbana del municipio de Cali, Colombia. Colombia Médica, 2007;38(004):386-394.

6. Alvarado SV, Suárez MC. Las transiciones escolares: una oportunidad de desarrollo integral para niños y niñas. Revista Latinoamericana de Ciencias Sociales, Niñez y Juventud, Manizales (julio-diciembre). 2009;7(2):907-928.

7. Gomes, AM. El proceso de escolarización de los Xakriabá: historia local y rumbos de la propuesta de educación escolar diferenciada. Cuad. Antropol. Soc. 2004;(19):29-48.

8. Rapiman DQ. Saberes y conocimientos indígenas en la formación de profesores de educación intercultural. Educ. Rev. 2007;(29):223-239.

\section{Correo electrónico de los autores}

Andrea Hernández Quirama: ahernanq@uis.edu.co, Blanca Patricia Mantilla Uribe: bpmantil@uis.edu.co, María Constanza Hakspiel Plata: mconina@yahoo.com, Claudia Milena Velasco Rangel: mile0833@hotmail.com, Maria del Pilar Oviedo: poviedoc@hotmail.com. 